\title{
Adsorption of atrazine on hemp stem-based activated carbons with different surface chemistry
}

\author{
Iwona Lupul $^{1} \cdot$ Jan $_{\text {Yperman }^{2}} \cdot$ Robert $_{\text {Carleer }}^{2} \cdot$ Grażyna Gryglewicz $^{1}$
}

Received: 2 April 2015/Revised: 13 July 2015/Accepted: 10 September 2015/Published online: 15 September 2015

(C) The Author(s) 2015. This article is published with open access at Springerlink.com

\begin{abstract}
Surface-modified hemp stem-based activated carbons (HACs) were prepared and used for the adsorption of atrazine from aqueous solution, and their adsorption performance was examined. A series of HACs were prepared by potassium hydroxide activation of hemp stems, followed by subsequent modification by thermal annealing, oxidation with nitric acid and amination. The resultant HACs differed in surface chemistry, while possessing similar porous structure. The surface group characteristics were examined by $\mathrm{X}$-ray photoelectron spectroscopy and measurement of the point of zero charge $\left(\mathrm{pH}_{\mathrm{PZC}}\right)$. The adsorption of atrazine from aqueous solution was performed in static conditions. The Langmuir-Freundlich and Langmuir models gave a better fit for equilibrium isotherms compared with the Freundlich model. The atrazine adsorption process was controlled by an intraparticle diffusion mechanism with a significant contribution from film diffusion. The presence of oxygen and nitrogen functionalities on the carbon surface was found to be undesirable for atrazine adsorption. The superior adsorbent was obtained by heat treatment of HAC in an inert atmosphere at $700{ }^{\circ} \mathrm{C}$, resulting in a very high adsorption capacity due to its enhanced hydrophobicity. The adsorption of atrazine on the studied HACs mainly involves $\pi-\pi$ dispersive
\end{abstract}

Grażyna Gryglewicz

grazyna.gryglewicz@pwr.edu.pl

1 Department of Polymer and Carbonaceous Materials, Faculty of Chemistry, Wrocław University of Technology, Gdańska 7/9, 50-344 Wrocław, Poland

2 Research Group of Applied and Analytical Chemistry, CMK, Hasselt University, Agoralaan Gebouw D, 3590 Diepenbeek, Belgium interactions between the atrazine ring and the graphene layers of carbon.

Keywords Hemp stem - Activated carbon - Surface chemistry $\cdot$ Adsorption $\cdot$ Atrazine

\section{Introduction}

Ground and drinking water contamination caused by agricultural pesticides is an environmental problem worldwide. The harmful effects of pesticides on human health and the environment have led to the adoption of stringent legislation concerning the purity of drinking water. EU Directive 98/83/EC sets the maximum concentration of single compounds and the total concentration of all pesticides in drinking water at 0.1 and $0.5 \mu \mathrm{g} / \mathrm{dm}^{3}$, respectively.

Atrazine (2-chloro-4-(ethylamino)-6-isopropylamino-striazine) is a common and selective triazine herbicide that is mostly used to control broadleaf and grass weeds on both agricultural and non-agricultural land (Mudhoo and Garg 2011). Although prohibited in the European Union since 2004, atrazine is still used in the cultivation of pineapple, macadamia nuts, corn, sorghum and sugarcane crops around the world (Ackerman 2007). Consequently, atrazine is frequently detected in groundwater, surface water and soils due to its high leaching potential and high chemical stability in soils and aqueous systems.

Adsorption on activated carbon (AC) is one of the most widely applied methods for the removal of organic contaminants from water (Rodríguez-Reinoso 1997; Bhatnagar and Sillanpää 2010). Commercial ACs are considered to be expensive due to the use of non-renewable and expensive materials such as coal. Therefore, the use of abundant and low-cost materials suitable for AC production, such as fruit 
stones, nut shells, cotton stalks and many others, has been intensively reported (Nor et al. 2013).

Hemp (Cannabis sativa L.) is a highly productive herbaceous plant with many industrial applications, including the production of paper, textiles, building materials, food, medicine, paint and fuel. Therefore, a substantial amount of waste is generated during hemp processing. The use of hemp-derived waste as precursor for AC manufacture could provide an attractive route for its disposal.

The performance of $\mathrm{AC}$ in the removal of organic compounds from water is strongly dependent on not only the adsorbent's porous texture but also its carbon surface chemistry and the characteristics of the adsorbate, i.e., its molecular dimensions, molar mass, solubility and $\mathrm{pK}_{\mathrm{a}}$ (Moreno-Castilla 2004). The processing conditions, such as solution $\mathrm{pH}$, ionic strength and temperature, are important as well (Terzyk 2000; Lorenc-Grabowska and Gryglewicz 2005, 2007). All of these factors influence the kinetics and mechanism of the adsorption of organic compounds on AC. The presence of oxygen and nitrogen functional groups on the carbon surface controls the surface properties of ACs. These functionalities determine the surface charge, the surface hydrophobicity, and the electron density of the graphene layers (Radovic et al. 2001). Oxygen-containing groups, such as carboxylic, phenolic and carbonyl/quinone groups, are the source of AC's acidic properties, whereas chromene- and pyrone-type structures are responsible for its enhanced surface basicity (Moreno-Castilla 2004; Radovic et al. 2001). Furthermore, nitrogen-containing groups, such as pyridine-type structures, pyridonic and amine/amide and lactam functionalities, also contribute to AC's basic properties. The concentration of oxygen and nitrogen functional groups on the carbon surface of AC can be enhanced through appropriate treatment. Wet oxidation using different oxidants $\left(\mathrm{HNO}_{3}, \mathrm{H}_{2} \mathrm{O}_{2},\left(\mathrm{NH}_{4}\right)_{2} \mathrm{~S}_{2} \mathrm{O}_{8}\right)$ is the most common approach used to incorporate oxygen groups into the AC surface (Moreno-Castilla et al. 2000). Nitrogen groups are predominantly introduced by reaction with ammonia and other nitrogen-containing compounds such as urea and melamine (Przepiórski et al. 2004; Seredych et al. 2008; Chen et al. 2012). In addition, the basicity of the carbon surface can also be associated with the delocalized $\pi$-electrons of the graphene layer (Radovic et al. 2001).

In the literature, little attention has been given to the adsorption of atrazine onto ACs with modified acidic and/ or basic surface properties. Chingombe et al. (2006) studied the effect of heat treatment of commercial AC (F400) in nitrogen and ammonia atmospheres on the removal of atrazine from aqueous solution. In a similar report by Mazet et al. (1994), the sorption performance of the same adsorbent was studied. It was found that high-temperature treatment of carbon samples in an inert atmosphere enhances atrazine uptake to a greater extent than amination. In both cases, the authors ignored the changes in the porous structure caused by AC modification. However, Pelekani and Snoeyink (2001) reported that the adsorption capacity of AC towards atrazine is strongly related to the volume of micropores with a width of $0.8-2 \mathrm{~nm}$. Therefore, there is a need to clarify the contribution of carbon surface chemistry to the overall adsorption of atrazine from aqueous solution. In this context, further elucidating the interactions between atrazine and $\mathrm{AC}$ with different surface properties is an important step for the development of highperformance adsorbents for atrazine removal. Atrazine can act as a $\pi$ electron donor and shows both $\mathrm{H}$-bond donor and acceptor properties (Welhouse and Bleam 1993). The H-bonding mechanism plays an important role in the sorption of atrazine by soils and natural sediments (Kovaios et al. 2006). Hydrophobic interactions have also been implicated in atrazine removal by multi-walled carbon nanotubes (MWCNTs) (Tang et al. 2012).

The aim of this study was to investigate the sorption performance of ACs with different surface properties but similar porous structure for the removal of atrazine from aqueous solution. For this purpose a series of microporous hemp stem-based activated carbons (HACs) was prepared by $\mathrm{KOH}$ activation, followed by subsequent modification by annealing at high temperature in a nitrogen atmosphere, oxidation with nitric acid and reaction with ammonia. To date, no study has been conducted on the use of HACs for the removal of atrazine from aqueous solution. The adsorption kinetics and equilibrium isotherms of atrazine sorption onto the surface of modified ACs were determined to obtain a better understanding of the mechanism of atrazine adsorption by clarifying the role of carbon surface chemistry.

\section{Experimental}

\subsection{Preparation of the initial AC}

Dry hemp ( $C$. sativa L.) stems were used in the preparation of ACs. Prior to use, the hemp stems were stripped of leaves, cut into lengths of approximately $5 \mathrm{~mm}$ and kept at room temperature. Carbonization of the precursor was performed in a horizontal tubular furnace heated to $600{ }^{\circ} \mathrm{C}$ at a heating rate of $10{ }^{\circ} \mathrm{C} / \mathrm{min}$ in a nitrogen atmosphere. Samples were held at the final temperature for $1 \mathrm{~h}$. After the carbonization step, the resultant char was physically mixed with anhydrous $\mathrm{KOH}$ at a 1:3 weight ratio. Then, the mixture was placed in a nickel boat and heated at a rate of $10{ }^{\circ} \mathrm{C} / \mathrm{min}$ to the final temperature of $700{ }^{\circ} \mathrm{C}$ under nitrogen flow and held at that temperature for $1 \mathrm{~h}$. The activated product was cooled to room temperature, washed with a 
$10 \%$ solution of hydrochloric acid, further washed with hot distilled water until reaching neutral $\mathrm{pH}$, and finally dried at $110{ }^{\circ} \mathrm{C}$ overnight. The resultant hemp stem-based AC was labeled as HAC and was used as a starting material to produce a series of samples with different surface chemistry but with no differences in their porous structure.

\subsection{Modifications of carbon surface}

The initial HAC was oxidized with $40 \% \mathrm{HNO}_{3}$ under reflux at boiling point for $7 \mathrm{~min}$ to enhance the acidic character of the carbon surface. After treatment, the sample was washed with distilled water until reaching neutral $\mathrm{pH}$ and dried overnight at $55{ }^{\circ} \mathrm{C}$ in a vacuum oven. This oxidized $\mathrm{AC}$ was labeled as $\mathrm{HAC}_{\mathrm{HNO}}$.

Another portion of the HAC was treated by gaseous $\mathrm{NH}_{3}$ to introduce nitrogen-containing surface groups which made the carbon surface basic. The amination process was performed in a horizontal quartz reactor at $700{ }^{\circ} \mathrm{C}$ under an ammonia flow of $150 \mathrm{~cm}^{3} / \mathrm{min}$ for $3 \mathrm{~h}$. Afterward, the product was cooled to a temperature of $200{ }^{\circ} \mathrm{C}$ in a flow of ammonia and subsequently cooled to room temperature in a flow of nitrogen. This $\mathrm{NH}_{3}$-treated sample was labeled as $\mathrm{HAC} \mathrm{NH}_{3}$.

Thermal treatment was also applied to produce AC with basic characteristics. Another HAC sample was placed in a quartz boat inside a horizontal tubular furnace and heated to $700{ }^{\circ} \mathrm{C}$ at $10{ }^{\circ} \mathrm{C} / \mathrm{min}$ under a nitrogen flow $\left(330 \mathrm{~cm}^{3} /\right.$ min) and maintained at this temperature for $1 \mathrm{~h}$. The resultant $\mathrm{AC}$ was labeled as $\mathrm{HAC} \mathrm{N}_{2}$.

\subsection{Characterization of ACs}

The elemental analysis of $\mathrm{C}, \mathrm{H}$ and $\mathrm{N}$ was performed using a Vario III Elemental Analyzer. The oxygen content was determined by a direct method using an EuroVector 3018 apparatus.

For the determination of the relative surface concentrations of carbon, oxygen and nitrogen functionalities, X-ray photoelectron spectroscopy (XPS) measurements were performed on an ESCALAB-210 spectrophotometer. A non-linear, Shirley-type baseline and an iterative leastsquare fitting algorithm were used for the deconvolution of the $\mathrm{C} 1 \mathrm{~s}$ and $\mathrm{N} 1 \mathrm{~s}$ spectra. A mixing ratio of $70 \%$ Gaussian and $30 \%$ Lorentzian functions was applied.

The porous structure parameters of the ACs were determined from nitrogen adsorption isotherms measured at $77 \mathrm{~K}$ with an ASAP2020 gas sorption analyzer (Micromeritics). Prior to measurements, samples were outgassed overnight at $300{ }^{\circ} \mathrm{C}$. The specific surface area $\left(\mathrm{S}_{\mathrm{BET}}\right)$ was calculated using the Brunauer-Emmett-Teller (BET) method. The amount of nitrogen adsorbed at a relative pressure of $\mathrm{p} / \mathrm{p}_{0}=0.96$ was employed to determine the total pore volume $\left(\mathrm{V}_{\mathrm{T}}\right)$. The micropore volume $\left(\mathrm{V}_{\mathrm{DR}}\right)$ and average micropore width $\mathrm{L}_{0}$ were determined by applying the Dubinin-Radushkevich and Stoeckli equations (Stoeckli et al. 1999). The micropore fraction was expressed as the ratio of the micropore volume to the total pore volume. Pore size distributions were calculated from the isotherm data by applying the quenched-solid density functional theory method (QSDFT) (Neimark et al. 2009).

The $\mathrm{pH}_{\mathrm{PZC}}$ (point of zero charge) of the ACs was determined by mixing $0.25 \mathrm{~g}$ of sample with $10 \mathrm{~cm}^{3}$ of $\mathrm{CO}_{2}$-free distilled water, according to the procedure described by Moreno-Castilla et al. (2000).

\subsection{Adsorption tests}

The adsorption of atrazine from aqueous solution was performed at $25{ }^{\circ} \mathrm{C}$ in a static system. A sieved fraction of AC between 0.5 and $1.0 \mathrm{~mm}$ was used for the sorption experiments. To establish the equilibrium time, 50-mg samples of AC were transferred into Erlenmeyer flasks, each containing $0.1 \mathrm{dm}^{3}$ of adsorbate solution $(30 \mathrm{mg} /$ $\mathrm{dm}^{3}$ ), which were kept in a thermostat shaker bath and agitated until equilibrium was achieved.

For determination of the adsorption isotherm, 5-100 mg of $\mathrm{AC}$ was placed into flasks and $0.1 \mathrm{dm}^{3}$ of atrazine solution $\left(30 \mathrm{mg} / \mathrm{dm}^{3}\right)$ was added to each of the flasks. An uniform shaking speed was maintained for all the experiments. Each set of flasks included two additional flasks containing a blank solution to account for the adsorption of sorbate on the glass walls. The adsorption isotherms were determined without adding any buffer to control $\mathrm{pH}$, to avoid the presence of a new electrolyte in the system. Before and after contact, the solution $\mathrm{pH}$ was measured using a digital pH-meter (Mettler Toledo) with a glass electrode. Each adsorption test was conducted in duplicate. The concentration of solute remaining in the water phase was measured using a HITACHI U-2800 UV-Vis spectrophotometer at a wavelength of $222 \mathrm{~nm}$. The basic physicochemical properties of atrazine are given in Table 1.

\section{Results and discussion}

\subsection{Characteristics of HACs}

This study demonstrates that by using different controlled methods of surface modification, it was possible to obtain a series of ACs characterized by different surface chemistry but similar porous structure. Table 2 summarizes the elemental composition, porous structure parameters and $\mathrm{pH}_{\mathrm{PZC}}$ for the various ACs. The studied HACs are characterized by comparable total pore volumes ranging from 
Table 1 Physicochemical characteristics of atrazine

\begin{tabular}{ll}
\hline Parameter & \multicolumn{3}{c}{ Structural formula } \\
\hline Molecular formula & $\mathrm{C}_{8} \mathrm{H}_{14} \mathrm{ClN}_{5}$ \\
Molar mass $(\mathrm{g} / \mathrm{mol})$ & 215.7 \\
Water solubility at $20{ }^{\circ} \mathrm{C}\left(\mathrm{mg} / \mathrm{dm}^{3}\right)$ & 30 \\
$\mathrm{pK}_{\mathrm{b}}$ & 12.3 \\
Molecule size $(\mathrm{nm})$ & 0.96 \\
Width $(\mathrm{nm})$ & 0.84 \\
Depth $(\mathrm{nm})$ & 0.544 \\
$\sigma(\mathrm{nm})^{2}$ & 0.832 \\
$\mathrm{~d}_{\mathrm{ef}}=2(\sigma / \pi)^{1 / 2}(\mathrm{~nm})$ &
\end{tabular}

${ }^{a}$ Cross-sectional area of atrazine molecule (Borisover and Graber 1997)

Table 2 Characteristics of hemp stem-based activated carbons

\begin{tabular}{|c|c|c|c|c|c|c|c|c|c|c|}
\hline \multirow[t]{2}{*}{ Activated carbon } & \multicolumn{4}{|c|}{ Elemental analysis (wt\%) } & \multicolumn{5}{|c|}{ Porous structure parameters } & \multirow[t]{2}{*}{$\mathrm{pH}_{\mathrm{PZC}}$} \\
\hline & $\mathrm{C}$ & $\mathrm{H}$ & $\mathrm{N}$ & $\mathrm{O}$ & $\mathrm{S}_{\mathrm{BET}}\left(\mathrm{m}^{2} / \mathrm{g}\right)$ & $\mathrm{V}_{\mathrm{T}}\left(\mathrm{cm}^{3} / \mathrm{g}\right)$ & $\mathrm{V}_{\mathrm{DR}}\left(\mathrm{cm}^{3} / \mathrm{g}\right)$ & $\mathrm{V}_{\mathrm{DR}} / \mathrm{V}_{\mathrm{T}}$ & $\mathrm{L}_{0}(\mathrm{~nm})$ & \\
\hline HAC & 84.0 & 1.9 & 0.3 & 13.8 & 2135 & 0.919 & 0.829 & 0.90 & 1.15 & 6.6 \\
\hline $\mathrm{HAC} \mathrm{N}_{2}$ & 90.3 & 0.4 & 0.5 & 8.8 & 2213 & 0.953 & 0.843 & 0.88 & 1.24 & 8.1 \\
\hline $\mathrm{HAC} \mathrm{NH}_{3}$ & 88.1 & 0.6 & 5.3 & 6.0 & 2088 & 0.927 & 0.785 & 0.85 & 1.36 & 9.2 \\
\hline 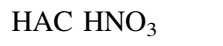 & 76.0 & 1.3 & 0.8 & 21.9 & 2067 & 0.910 & 0.781 & 0.86 & 1.30 & 3.9 \\
\hline
\end{tabular}

0.910 to $0.953 \mathrm{~cm}^{3} / \mathrm{g}$ and a BET surface area between 2067 and $2213 \mathrm{~m}^{2} / \mathrm{g}$. Micropores constitute over $85 \%$ of the total pore volume. The average micropore size $\mathrm{L}_{0}$ is in the range of $1.15-1.36 \mathrm{~nm}$. Similarity in the porous structure for HACs is confirmed by the pore size distribution determined by the QSDFT method (Fig. 1).

The heat treatment of HAC in nitrogen and ammonia atmospheres results in an increase of carbon content followed by a decrease of oxygen content and renders the carbon surface more basic (Table 2). $\mathrm{N}_{2}$ treatment at $700{ }^{\circ} \mathrm{C}$ removes acidic oxygen functional groups to a large extent, which leads to enhanced $\pi$ electron density in the

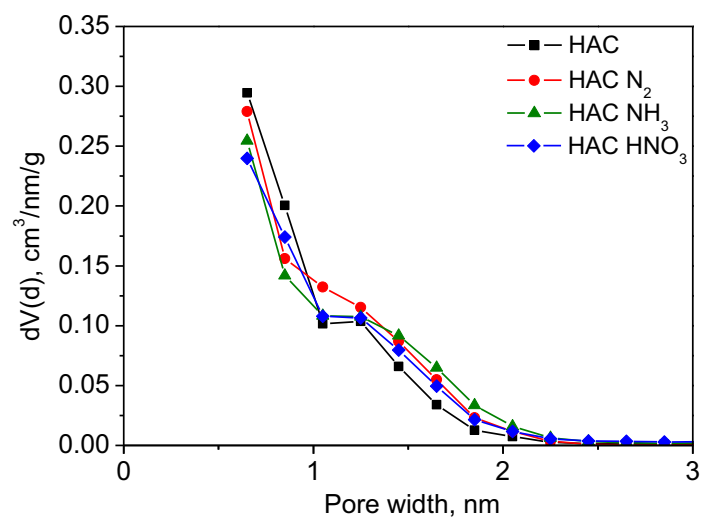

Fig. 1 Pore size distribution of HACs determined by OSDFT method graphene layer. During ammonia treatment, a substantial amount of nitrogen was introduced into the carbon matrix (5.3 vs $0.3 \mathrm{wt} \%$ ). As a result of both treatments, the $\mathrm{pH}_{\mathrm{PZC}}$ increased from 6.6 to 8.1 and 9.2, respectively, for $\mathrm{HAC} \mathrm{N}_{2}$ and $\mathrm{HAC} \mathrm{NH}_{3}$. The oxidation of $\mathrm{HAC}$ with nitric acid leads to an increase in the oxygen content from 13.8 to $21.9 \mathrm{wt} \%$ and generates acidic carbon surface properties $\left(\mathrm{pH}_{\mathrm{PZC}}=3.9\right)$. Notably, even a short 7-min oxidative treatment resulted in a significant enhancement of oxygen content, most likely due to the highly developed surface area of the initial HAC carbon.

XPS analysis was used to determine the functional groups on the surface of oxygen- and nitrogen-rich ACs. Table 3 contains the carbon, nitrogen and oxygen surface concentrations of the initial and modified HACs together with the distribution of functionalities derived from the $\mathrm{C} 1 \mathrm{~s}$ spectrum. The $\mathrm{C} 1 \mathrm{~s}$ signal was deconvoluted into five components that represent carbon atoms in $\mathrm{C}-\mathrm{C}$ nonfunctional graphitic structures $(284.8 \pm 0.1 \mathrm{eV})$; carbon present in phenolic, alcohol and ether groups (286.7 \pm $0.3 \mathrm{eV})$; carbonyl and quinone groups $(287.6 \pm 0.2 \mathrm{eV})$; carboxyl and lactone groups $(288.9 \pm 0.3 \mathrm{eV})$ and a shakeup satellite peak due to $\pi-\pi^{*}$ transitions in aromatic rings (290.4 $\pm 0.4 \mathrm{eV}$ ) (Biniak et al. 1997).

The changes in the surface concentration of carbon, nitrogen and oxygen (Table 3) induced by thermal annealing, amination and oxidative treatment are consistent 
Table 3 Surface carbon, nitrogen and oxygen contents and relative concentration of functional groups obtained from C1s XPS spectra for HACs

\begin{tabular}{lrrrr}
\hline & $\mathrm{HAC}$ & $\mathrm{HAC} \mathrm{N}_{2}$ & $\mathrm{HAC} \mathrm{NH}_{3}$ & ${\mathrm{HAC} \mathrm{HNO}_{3}}$ \\
\hline Surface content (at.\%) & & & & \\
C & 86.1 & 92.7 & 90.4 & 82.5 \\
N & 0.6 & 0.6 & 4.2 & 0.8 \\
O & 13.3 & 4.2 & 5.4 & 17.7 \\
Relative surface concentration (\%) & & & & \\
C-C (aromatic and aliphatic) & 74 & 76 & 74 & 68 \\
Single C-O (C-OH, C-O-C) & 11 & 9 & 11 & 12 \\
C = O, C-N & 7 & 7 & 8 & 9 \\
O-C = O & 4 & 3 & 3 & 3 \\
$\pi-\pi *$ shake-up satellite & 4 & 5 & 4 & 3 \\
\hline
\end{tabular}

with those determined for the bulk material by elemental analysis (Table 2). The carbon content on the surface is the highest for $\mathrm{HAC} \mathrm{N}_{2}$. An increase in the surface nitrogen content from 0.6 to 4.2 at. \% can be observed for $\mathrm{HAC} \mathrm{NH}_{3}$. As expected, $\mathrm{HAC} \mathrm{HNO}_{3}$ is characterized by the highest surface oxygen content (17.7 at.\%) among the studied carbons. However, the elemental contents on the surface of the HACs are different from those of the bulk material. This variation in elemental content can be explained by the different characteristics of the carbon surfaces and the overall characteristics of each material's bulk properties.

The carbon atoms in $\mathrm{C}-\mathrm{C}$ graphitic structures constitute $74-76 \%$ of the total carbon content in the HAC, HAC $\mathrm{N}_{2}$ and $\mathrm{HAC} \mathrm{NH}_{3}$ carbons. A significantly lower contribution

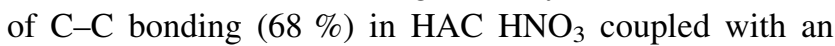
enhanced amount of carbon atoms in oxygen-containing functional groups. HAC $\mathrm{HNO}_{3}$ carbon is distinguished among the studied HACs by a more than two fold increase in carboxylic groups; these groups are mainly responsible for the high acidity of the surface (Table 2).

Figure 2 shows the deconvolution of the N1s region of the XPS spectrum for $\mathrm{HAC} \mathrm{NH}_{3}$ in order to determine

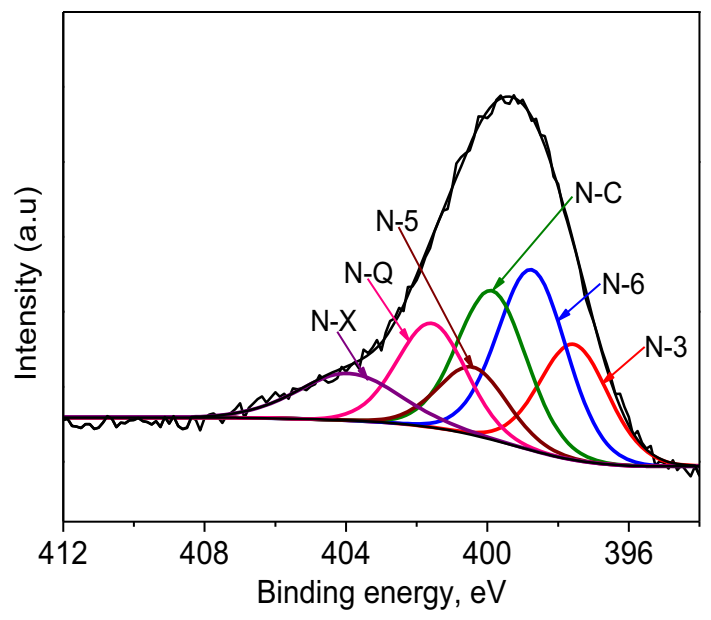

Fig. 2 N1s XPS spectra of HAC treated with ammonia nitrogen-containing surface groups. The N1s signal has been fitted by six components with binding energies of $397.7 \pm 0.3 \mathrm{eV}$ (nitrile $\mathrm{N}-3$ ); $398.7 \pm 0.3 \mathrm{eV}$ (pyridinic $\mathrm{N}-6) ; \quad 399.7 \pm 0.2 \mathrm{eV}$ (amide, imide, lactame $\mathrm{N}-\mathrm{C}$ ); $400.3 \pm 0.3 \mathrm{eV}$ (pyrrolic/pyridonic N-5); $401.4 \pm 0.3 \mathrm{eV}$ (quaternary nitrogen N-Q) and 402-405 eV (pyridine-Noxide N-X) (Lorenc-Grabowska et al. 2013). Almost half of the surface nitrogen occurs in pyridinic and amide, imide, lactame structures in the aminated carbon. Basic pyridonic groups also may contribute to the carbon basicity. This explains the highly basic character of the HAC $\mathrm{NH}_{3}$ carbon surface.

\subsection{Adsorption of atrazine from aqueous solution}

\subsubsection{Kinetics of adsorption}

The amount of atrazine adsorbed versus contact time for the HACs studied is shown in Fig. 3. The atrazine adsorption processes monitored here are fast. With the exception of $\mathrm{HAC} \mathrm{HNO}_{3}$, over $80 \%$ of the equilibrium sorption capacity is attained after the first $1 \mathrm{~h}$. The time needed to reach equilibrium in atrazine uptake increases as follows: $\mathrm{HAC} \mathrm{N}_{2}$, $\mathrm{HAC} \mathrm{NH}_{3}(2 \mathrm{~h})<\mathrm{HAC}(4 \mathrm{~h})<\mathrm{HAC} \mathrm{HNO}_{3}(5 \mathrm{~h})$. It was

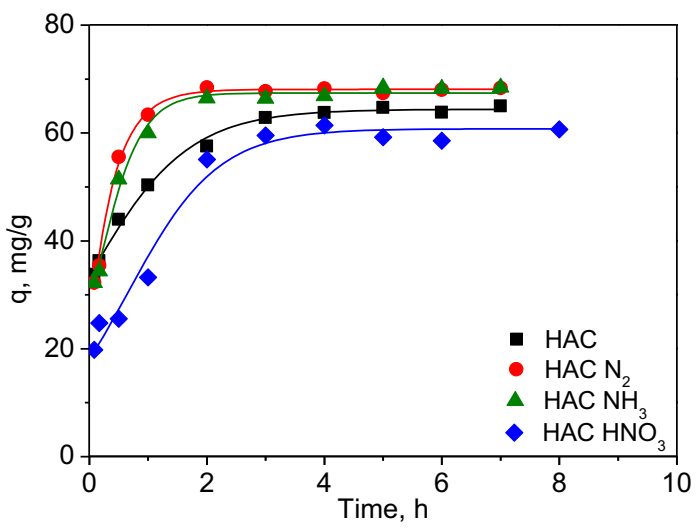

Fig. 3 Effect of contact time on the adsorbed amount of atrazine 
revealed that with increased hydrophilicity of the carbon surface, the time to reach equilibrium becomes longer. HAC $\mathrm{HNO}_{3}$ carbon has the most hydrophilic nature among the studied HACs due to the presence of a large amount of surface oxygen functionalities, mainly hydroxylic and carboxylic groups (Table 3). As a consequence, water molecules, which are preferentially adsorbed on the polar sites of the carbon surface, block the entrance of the adsorbent pores and make adsorption more difficult (Wang et al. 2006). Chen et al. (2009) also reported that atrazine's adsorption affinity towards the carbon surface decreases with increased oxygen content despite the similar mesoporous structural parameters of the MWCNTs used in the study.

Taking into account that adsorption kinetics is controlled by diffusion mechanism, the intraparticle diffusion model based on the theory proposed by Weber and Morris (1963) was used to determine the rate-limiting step of the adsorption process. The intraparticle diffusion model is expressed by the following empirical equation (Weber and Morris 1963):

$q_{t}=k_{i d} t^{1 / 2}+C$

where $\mathrm{q}_{\mathrm{t}}$ and $\mathrm{k}_{\mathrm{id}}$ are the amount of atrazine adsorbed at time $\mathrm{t}(\mathrm{mg} / \mathrm{g})$ and the intraparticle rate constant $[\mathrm{mg} /$ $\left.\left(\mathrm{g} \min ^{1 / 2}\right)\right]$, respectively. $\mathrm{C}[\mathrm{mg} / \mathrm{g}]$ is the intercept, and it represents an external resistance for mass transfer from the bulk of solution across the boundary layer to the adsorbent surface. A larger $\mathrm{C}$ implies a greater boundary layer effect. The calculated values of $\mathrm{k}_{\mathrm{id}}$ and $\mathrm{C}$ are given in Table 4 .

To date, no comprehensive information is available on the application of the intraparticle diffusion model for the description of atrazine adsorption onto AC. Chen et al. (2009) reported that intraparticle diffusion was not the sole rate-controlling step of atrazine adsorption on oxidized MWCNTs. Chingombe et al. (2006) applied the Boyd kinetics equation to identify the slowest step of the process and concluded that the adsorption of atrazine onto modified ACs is controlled by a film diffusion mechanism. Figure 4 shows the Weber and Morris plots for atrazine uptake on the studied HACs. In our study, the plots are not linear over the whole time range and do not pass through the origin, indicating that more than one process affects atrazine adsorption. The intercept of the plot determines the contribution of

Table 4 Parameters of intraparticle diffusion kinetic model for atrazine adsorption on HACs

\begin{tabular}{llll}
\hline Adsorbent & $\mathrm{k}_{\mathrm{id}}\left(\mathrm{mg} / \mathrm{g} \mathrm{min}^{1 / 2}\right)$ & $\mathrm{C}(\mathrm{mg} / \mathrm{g})$ & $\mathrm{R}^{2}$ \\
\hline $\mathrm{HAC}$ & 2.62 & 28.68 & 0.993 \\
$\mathrm{HAC} \mathrm{N}$ & 6.05 & 18.40 & 0.964 \\
$\mathrm{HAC} \mathrm{NH}$ & 5.37 & 19.39 & 0.986 \\
$\mathrm{HAC} \mathrm{HNO}_{3}$ & 3.72 & 9.59 & 0.942 \\
\hline
\end{tabular}

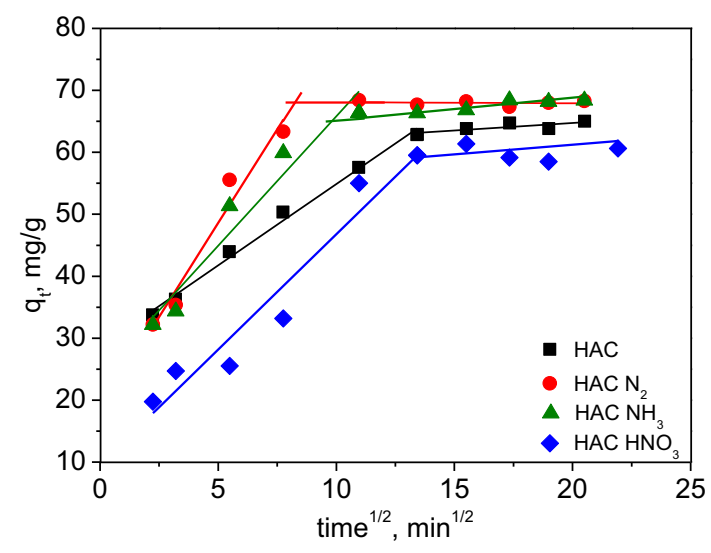

Fig. 4 Intraparticle diffusion model for the adsorption of atrazine on HACs

external layer diffusion in the atrazine adsorption process. The $\mathrm{C}$ value varies for the studied carbons as follows: $\mathrm{HAC}>\mathrm{HAC} \mathrm{NH}_{3}>\mathrm{HAC} \mathrm{N}_{2}>\mathrm{HAC} \mathrm{HNO}_{3}$ (Table 4). Except for the latter carbon, the decrease in the $\mathrm{C}$ value is related to decreasing heteroatom content. The lowest boundary layer effect for $\mathrm{HAC}_{\mathrm{HNO}}$ can be explained by its oxygen-enriched carbon surface, particularly in carboxylic group, which preferentially adsorb water molecules ( $\mathrm{Li}$ et al. 2002; Franz et al. 2000). Atrazine molecules undergo hydration in aqueous solution, which promotes their movement to hydrophilic carbon surface oxygen groups and the formation of clusters on the external surface of adsorbent, thus decreasing the boundary layer effect. The slope of the first part of the plot varies for the studied carbons and is the highest for $\mathrm{HAC} \mathrm{N}_{2}$, which reflects the fastest diffusion of atrazine molecules through mesopores and wider micropores to adsorption sites. Notably, $\mathrm{HAC} \mathrm{N}_{2}$ is characterized by both the lowest content of heteroatom-containing functional groups and the shortest equilibrium time among the studied HACs. The slope of the second part of the plot, which corresponds to diffusion in small micropores, is similar for all the HACs; this value, however, is significantly lower than that corresponding to diffusion in mesopores and wider micropores. This result indicates that intraparticle diffusion into the smaller micropores is the rate-limiting step in the process of atrazine adsorption on HACs.

\subsubsection{Equilibrium adsorption of atrazine}

Knowledge of the equilibrium adsorption isotherm is not only important to understand how adsorbate molecules interact with the adsorbent surface, but it is also critical in optimizing the use of $\mathrm{AC}$ as an adsorbent. The isotherms, i.e. experimental and theoretical, for the adsorption of atrazine by HACs are shown in Fig. 5. Except for HAC N 2 , the adsorption isotherms show a distinct plateau at higher 

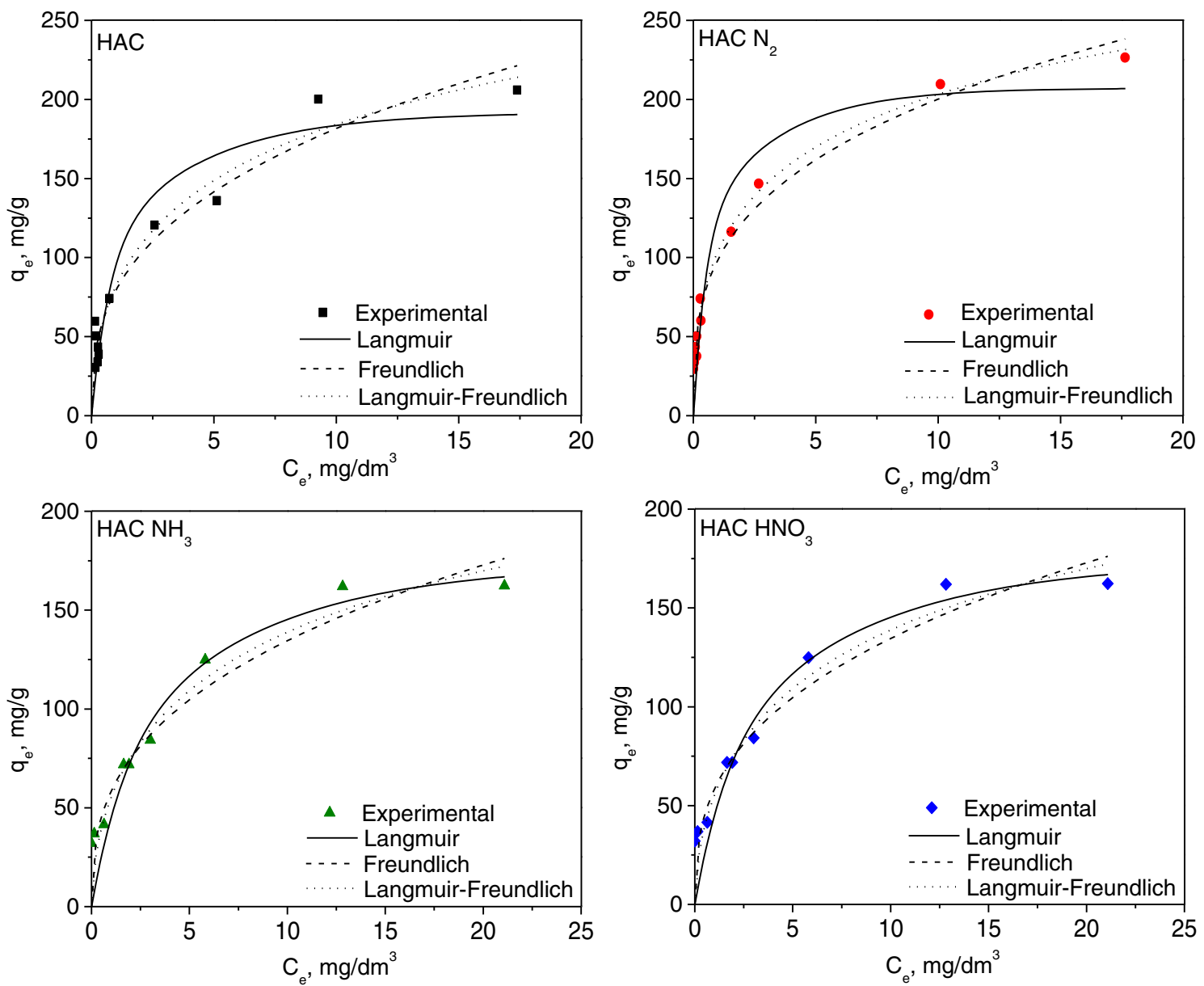

Fig. 5 Comparison of the experimental data and model fits of the Langmuir, Freundlich and Langmuir-Freundlich isotherms for the adsorption of atrazine on HACs

equilibrium concentrations for all studied HACs, corresponding to L-type isotherms according to the Giles classification. It is interesting to note the changes in the affinity of atrazine towards the surface of the HACs, which occur

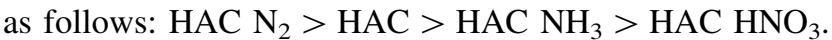
The lowest affinity of $\mathrm{HAC} \mathrm{HNO}_{3}$ among the studied carbons can be explained by the preferential adsorption of water molecules at low adsorptive concentration on carboxylic acid groups, which are abundant in this carbon (Table 3). In turn, the relatively low affinity of ammoniatreated HAC for atrazine is attributed to the enhanced amount of nitrogen functionalities, which favor bonding water molecules at a low equilibrium concentration. A study of PAN-based ACs showed that the hydrophobicity of the carbon surface decreases with increased nitrogen content (Lorenc-Grabowska et al. 2013).

The equilibrium adsorption isotherms were interpreted following the Langmuir (Eq. 2) (Langmuir 1918), Freundlich (Eq. 3) (Freundlich and Helle 1939) and LangmuirFreundlich (Eq. 4) (Marczewski et al. 1988) models: $q_{e}=\left(q_{\max } b C_{e}\right) /\left(1+\left(b C_{e}\right)\right)$

$q_{e}=K_{F} C_{e}^{1 / n}$

$q_{e}=q_{\max }\left(\left(K_{L F} C_{e}\right)^{n} /\left(1+\left(K_{L F} C_{e}\right)^{n}\right)\right)$

where $\mathrm{q}_{\mathrm{e}}$ is the amount of atrazine adsorbed by $\mathrm{AC}$ at equilibrium $(\mathrm{mg} / \mathrm{g}), \mathrm{C}_{\mathrm{e}}$ is the atrazine concentration equilibrium in solution $\left(\mathrm{mg} / \mathrm{dm}^{3}\right), \mathrm{q}_{\max }$ is the monolayer capacity of the adsorbent $(\mathrm{mg} / \mathrm{g}), \quad \mathrm{b}$ is the Langmuir adsorption constant $\left(\mathrm{dm}^{3} / \mathrm{mg}\right), \mathrm{K}_{\mathrm{F}}$ is the Freundlich constant $\left(\mathrm{mg}^{1-\mathrm{n}} \mathrm{dm}^{3 \mathrm{n}} / \mathrm{g}\right), \mathrm{K}_{\mathrm{LF}}$ is the Langmuir-type equilibrium constant defined by the Van't Hoff equation for a heterogeneous solid, and $\mathrm{n}$ is the Freundlich heterogeneity factor (the larger the value, the more heterogeneous the system). The calculated values of the Langmuir, Freundlich and Langmuir-Freundlich equations' parameters for atrazine adsorption are given in Table 5. Comparing the correlation coefficients of the three equations indicates that the equilibrium experimental data for HAC and HAC $\mathrm{N}_{2}$ are best fitted by the Langmuir-Freundlich equation, while 
atrazine adsorption onto $\mathrm{HAC} \mathrm{HNO}_{3}$ and $\mathrm{HAC} \mathrm{NH}_{3}$ is described by the Langmuir equation. Langmuir monolayer adsorption was also reported for atrazine adsorption onto activated carbon prepared from rubber tire waste (Gupta et al. 2011) and commercial activated carbon fibers (Faur et al. 2005). However, in most reports the sorption isotherms of atrazine for various adsorbents were best fitted by the Freundlich model (Kovaios et al. 2006; Tang et al. 2012; Labeau et al. 1999). For the studied HACs, the maximum adsorption capacity is clearly enhanced by the thermal annealing of HAC in a nitrogen atmosphere $(466 \mathrm{mg} / \mathrm{g}$ ). This result suggests that the $\pi-\pi$ dispersion interactions between the atrazine $\pi$ electron ring and the $\pi$ electrons of graphene layers play a dominant role in atrazine adsorption. The adsorption capacity is significantly reduced by amination and oxidative treatment of HAC (Table 5). Oxidized MWCNTs were also reported to perform worse than pristine MWCNTs in atrazine removal (Chen et al. 2009). The calculated parameter $n$ from the Langmuir-Freundlich equation indicates that HAC and HAC $\mathrm{N}_{2}$ have a more homogenous surface $(\mathrm{n}<0.50)$ than $\mathrm{HAC} \mathrm{HNO}_{3}$ and $\mathrm{HAC} \mathrm{NH}_{3}$ (Table 5). A less heterogeneous adsorbent surface seems to favor atrazine adsorption from aqueous solution. Considering the superior behavior of $\mathrm{HAC} \mathrm{N}_{2}$ in the adsorption of atrazine, an AC with a highly basic surface but free of heteroatom-containing surface functional groups is the most favorable for the removal of atrazine from aqueous solution. This finding is in agreement with the observed enhanced adsorption of atrazine by activated carbon annealed in a hydrogen flow (Chingombe et al. 2006).

\subsubsection{Mechanism of atrazine adsorption}

Many interactions, such as electrostatic interactions (Salvestrini et al. 2010), hydrophobic effects and van der Waals forces (Tang et al. 2012; Kulikova and Perminova 2002), $\pi-\pi$ interactions (Zhao et al. 2013), or hydrogen bonding (Welhouse and Bleam 1993; Kovaios et al. 2006) have been reported to be involved in atrazine adsorption on various adsorbents. Atrazine is a weakly basic herbicide with a $\mathrm{pK}_{\mathrm{b}}$ of 12.3 (Table 1). In our study, all adsorption processes were performed in a solution $\mathrm{pH}$ between 5 and 9. Under these conditions atrazine is adsorbed on the carbon surface as uncharged species, and as a result, electrostatic interactions may not influence its adsorption from aqueous solution. A study by Welhouse and Bleam (1993) emphasized the high hydrogen-bonding potential of atrazine molecules. In the triazine ring, the para nitrogen is a site where atrazine accepts a hydrogen bond, whereas the ethylamino side chain can be a hydrogen-bond donor. The ability of atrazine to form hydrogen bonds with the carboxyl and phenolic groups of humic substances was reported by Davies and Jabeen (2003). However, our study has indicated that no hydrogen bonding occurs between the adsorbed atrazine and oxygen and/or nitrogen functional groups on the carbon surface. If atrazine uptake was mainly governed by hydrogen bond formation, an S-type isotherm would be obtained, whereas an L-type isotherm is characteristic for the HACs studied. Moreover, if hydrogen bonding was a dominant mechanism of atrazine adsorption,

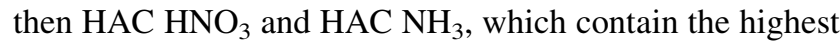
oxygen and nitrogen contents, respectively, should show the highest adsorption capacity. The acid carboxylic groups on the surface of oxidized HAC and pyridinic, pyridonic and amide/lactam groups in aminated HAC are the most likely places where a strong effect of the hydrogen bonding mechanism would be observed (Welhouse and Bleam 1993). On the contrary, a significant decrease in atrazine uptake by oxygen- and nitrogen-rich HACs was revealed. Presumably, the water molecules adsorb through hydrogen bonding on polar surface functional groups, forming clusters at the entrances of adsorbent pores and reducing the uptake of atrazine, particularly, at low adsorptive concentrations.

The obtained results show that the adsorption of atrazine is favored on annealed AC with basic properties. This finding emphasizes the importance of $\pi-\pi$ interactions between the aromatic $\mathrm{C}$ of graphene layers in the $\mathrm{AC}$ and the heterocyclic ring of atrazine. In the case of HAC $\mathrm{HNO}_{3}$, introducing electron-withdrawing oxygen functional groups reduces the electron density of graphene layers, decreasing $\pi-\pi$ interactions, which leads to a lower atrazine uptake. Like surface oxygen groups, the presence

Table 5 Langmuir, Freundlich and Langmuir-Freundlich parameters for atrazine adsorption on ACs

\begin{tabular}{|c|c|c|c|c|c|c|c|c|c|c|}
\hline \multirow[t]{2}{*}{ Adsorbent } & \multicolumn{3}{|l|}{ Langmuir } & \multicolumn{3}{|l|}{ Freundlich } & \multicolumn{4}{|c|}{ Langmuir-Freundlich } \\
\hline & $\mathrm{q}_{\max }(\mathrm{mg} / \mathrm{g})$ & $\mathrm{b}\left(\mathrm{dm}^{3} / \mathrm{g}\right)$ & $\mathrm{R}^{2}$ & $\mathrm{~K}_{\mathrm{F}}\left(\mathrm{mg}^{1-\mathrm{n}} \mathrm{dm}^{3 \mathrm{n}} / \mathrm{g}\right)$ & $1 / \mathrm{n}$ & $\mathrm{R}^{2}$ & $\mathrm{q}_{\max }(\mathrm{mg} / \mathrm{g})$ & $\mathrm{K}_{\mathrm{LF}}\left(\mathrm{dm}^{3} / \mathrm{mg}\right)$ & $\mathrm{n}$ & $\mathrm{R}^{2}$ \\
\hline HAC & 227 & 0.64 & 0.957 & 75 & 0.37 & 0.890 & 437 & 0.23 & 0.49 & 0.988 \\
\hline $\mathrm{HAC} \mathrm{N} 2$ & 263 & 0.90 & 0.967 & 96 & 0.31 & 0.948 & 466 & 0.29 & 0.43 & 0.988 \\
\hline $\mathrm{HAC} \mathrm{NH} 3$ & 179 & 0.50 & 0.974 & 59 & 0.34 & 0.933 & 317 & 0.21 & 0.57 & 0.916 \\
\hline 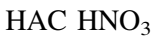 & 169 & 0.28 & 0.988 & 41 & 0.48 & 0.903 & 167 & 0.29 & 0.97 & 0.971 \\
\hline
\end{tabular}


of some nitrogen-containing functional groups on the carbon surface does not favour $\pi-\pi$ interactions between AC and atrazine. Radovic et al. (1997) came to the same conclusion for nitrobenzene and aniline adsorption on oxidized and aminated carbons. When the adsorption process is governed by $\pi-\pi$ interactions, reduced adsorption is the consequence of withdrawal and/or localization of $\pi$ electrons caused by carbon oxidation and amination.

Nevertheless, hydrophobic interactions cannot be excluded in the adsorption of atrazine on HACs. As previously mentioned, polar oxygen and nitrogen groups on the carbon surface can act as water binding centers and facilitate the formation of water clusters, which may reduce the accessibility of adsorption sites to atrazine because of atrazine's hydrophobicity (Chingombe et al. 2006). Considering that HAC $\mathrm{N}_{2}$ is characterized by the highest adsorption capacity among the studied HACs, the increased hydrophobicity of the AC surface caused by removing acidic functionalities may be a key factor for the excellent adsorption of atrazine from aqueous solution.

\section{Conclusions}

The present work has demonstrated the important role of surface chemistry in the adsorption of atrazine onto HACs. Atrazine uptake increased with a decreasing amount of oxygen- and nitrogen-containing groups on the carbon surface. This result indicates that the dominant mechanism for the adsorption of atrazine involves $\pi-\pi$ dispersive interactions between the graphene layers of the adsorbent and the heterocyclic ring of atrazine. The adsorption kinetics study shows that the atrazine adsorption process is controlled by intraparticle diffusion with a reasonable contribution from boundary layer diffusion. Intraparticle diffusion into small micropores is found to be the ratelimiting step in atrazine adsorption.

Adsorption equilibrium data were best described by the Langmuir and Langmuir-Freundlich isotherm models. The highest adsorption capacity was obtained for $\mathrm{HAC} \mathrm{N}_{2}$, which is characterized by a basic character and the lowest amount of heteroatoms among the studied HACs. The annealing of $\mathrm{AC}$ results in a reduced amount of oxygen functional groups and consequently enhances carbon surface hydrophobicity and the $\pi$ electron density of the graphene layers.

The obtained results indicate that a hydrophobic carbon surface promotes atrazine adsorption. Strong polar sites on the carbon surface such as carboxylic, pyridinic, pyridonic and amide/lactam groups decreased the adsorption capacity to a high extent. The substantially lower adsorption capacities of $\mathrm{HAC} \mathrm{HNO}_{3}$ and $\mathrm{HAC} \mathrm{NH}_{3}$ prove that porous carbons rich in oxygen and/or nitrogen are not suitable for the removal of atrazine from aqueous solution. The annealing of $\mathrm{AC}$ is therefore recommended as a way to improve atrazine adsorption.

Acknowledgments This work was financed by a statutory activity subsidy from the Polish Ministry of Science and Higher Education for the Faculty of Chemistry of Wrocław University of Technology.

Open Access This article is distributed under the terms of the Creative Commons Attribution 4.0 International License (http://crea tivecommons.org/licenses/by/4.0/), which permits unrestricted use, distribution, and reproduction in any medium, provided you give appropriate credit to the original author(s) and the source, provide a link to the Creative Commons license, and indicate if changes were made.

\section{References}

Ackerman, F.: The economics of atrazine. Int. J. Occup. Environ. Health 13, 437-445 (2007)

Bhatnagar, A., Sillanpää, M.: Utilization of agro-industrial and municipal waste materials as potential adsorbents for water treatment-a review. Chem. Eng. J. 157, 277-296 (2010)

Biniak, S., Szymański, G., Siedlewski, J., Świątkowski, A.: The characterization of activated carbon with oxygen and nitrogen surface groups. Carbon 35, 1799-1810 (1997)

Borisover, M.D., Graber, E.R.: Comment to "Competitive sorption between atrazine and other organic compounds in soils and model sorbents". Environ. Sci. Technol. 31, 1577 (1997)

Chen, G.-C., Shan, X.-Q., Zhou, Y.-Q., Shen, X., Huang, H.-L., Khan, S.U.: Adsorption kinetics, isotherms and thermodynamics of atrazine on surface oxidized multiwalled carbon nanotubes. J. Hazard. Mater. 169, 912-918 (2009)

Chen, J., Zhai, Y., Chen, H., Li, C., Zeng, G., Pang, D., Lu, P.: Effects of pretreatment on the surface chemistry and pore size properties of nitrogen functionalized and alkylated granular activated carbon. Appl. Surf. Sci. 263, 247-253 (2012)

Chingombe, P., Saha, B., Wakeman, R.J.: Sorption of atrazine on conventional and surface modified activated carbons. J. Colloid Interface Sci. 302, 408-416 (2006)

Davies, J.E.D., Jabeen, N.: The adsorption of herbicides and pesticides on clay minerals and soils. Part 2. Atrazine. J. Incl. Phenom. Macrocycl. Chem. 46, 57-64 (2003)

Faur, C., Métivier-Pignon, H., Le Cloirec, P.: Multicomponent adsorption of pesticides onto activated carbon fibers. Adsorption 11, 479-490 (2005)

Franz, M., Arafat, H.A., Pinto, N.G.: Effect of chemical surface heterogeneity on the adsorption mechanism of dissolved aromatics on activated carbon. Carbon 38, 1807-1819 (2000)

Freundlich, H., Helle, W.J.: Ubber die adsorption in lusungen. J. Am. Chem. Soc. 61, 2-28 (1939)

Gupta, V.K., Gupta, B., Rastogi, A., Agarwal, S., Nayak, A.: Pesticides removal from waste water by activated carbon prepared from waste rubber tire. Water Res. 45, 4047-4055 (2011)

Kovaios, I.D., Paraskeva, C.A., Koutsoukos, P.G., Payatakes, A.Ch.: Adsorption of atrazine on soils: model study. J. Colloid Interface Sci. 299, 88-94 (2006)

Kulikova, N.A., Perminova, I.V.: Binding of atrazine to humic substances from soil, peat and coal related to their structure. Environ. Sci. Technol. 36, 3720-3724 (2002)

Labeau, T., Lelièvre, C., Wolbert, D., Laplanche, A., Prados, M., Côté, P.: Effect of natural organic matter loading on the atrazine 
adsorption capacity of an aging powdered activated carbon slurry. Water Res. 33, 1695-1705 (1999)

Langmuir, I.: The adsorption of gases in plain surface of glass, mica, and platinum. J. Am. Chem. Soc. 40, 1361-1403 (1918)

Li, L., Quinlivan, P.A., Knappe, D.R.U.: Effects of activated carbon surface chemistry and pore structure on the adsorption of organic contaminants from aqueous solution. Carbon 40, 2085-2100 (2002)

Lorenc-Grabowska, E., Gryglewicz, G.: Adsorption of lignite-derived humic acid on coal-based mesoporous activated carbons. J. Colloid Interface Sci. 284, 416-423 (2005)

Lorenc-Grabowska, E., Gryglewicz, G.: Adsorption characteristics of Congo Red on coal-based mesoporous activated carbon. Dyes Pigments 74, 34-40 (2007)

Lorenc-Grabowska, E., Gryglewicz, G., Diez, M.A.: Kinetics and equilibrium study of phenol adsorption on nitrogen-enriched activated carbons. Fuel 114, 235-243 (2013)

Marczewski, A.W., Deryło-Marczewska, A., Jaroniec, M.: Correlations of heterogeneity parameters for single-solute and multisolute adsorption from dilute solutions. J. Chem. Soc. Faraday Trans. 84, 2951-2957 (1988)

Mazet, M., Farkhani, B., Baudu, M.: Influence of heat or chemical treatment of activated carbon onto the adsorption of organic compounds. Water Res. 28, 1609-1617 (1994)

Moreno-Castilla, C.: Adsorption of organic molecules from aqueous solutions on carbon materials. Carbon 42, 83-94 (2004)

Moreno-Castilla, C., Lopez-Ramon, M.V., Carrasco-Marin, F.: Changes in surface chemistry of activated carbons by wet oxidation. Carbon 38, 1995-2001 (2000)

Mudhoo, A., Garg, V.K.: Sorption, transport and transformation of atrazine in soils, minerals and composts: a review. Pedosphere 21, 11-25 (2011)

Neimark, A.V., Lin, Y., Ravikovitch, P.I., Thommes, M.: Quenched solid density functional theory and pore size analysis of micromesoporous carbons. Carbon 47, 1617-1628 (2009)

Nor, M.N., Chung, L.L., Teong, L.K., Mohamed, A.R.: Synthesis of activated carbon from lignocellulosic biomass and its application in air pollution control-a review. J. Environ. Chem. Eng. 1, 658-666 (2013)

Pelekani, C., Snoeyink, V.L.: A kinetic and equilibrium study of competitive adsorption between atrazine and Congo red dye on activated carbon: the importance of pore size distribution. Carbon 39, 35-37 (2001)

Przepiórski, J., Skrodzewicz, M., Morawski, A.W.: High temperature ammonia treatment of activated carbon for enhancement of $\mathrm{CO}_{2}$ adsorption. Appl. Surf. Sci. 225, 235-242 (2004)
Radovic, L.R., Silva, I.F., Ume, J.I., Menendez, J.A., Leon, Y., Leon, C.A., Scaroni, A.W.: An experimental and theoretical study of the adsorption of aromatics possessing electron-withdrawing and electron-donating functional groups by chemically modified activated carbon. Carbon 35, 1339-1348 (1997)

Radovic, L.R., Moreno-Castilla, C., Rivera-Utrilla, J.: Carbon materials as adsorbents in aqueous solution. In: Radovic, L.R. (ed.) Chemistry and Physics of Carbon. vol 27, pp. 228-405. Marcel Dekker, New York (2001)

Rodríguez-Reinoso, F.: Activated carbon: structure, characterization, preparation and applications. In: Marsh, H., Heintz, E.A., Rodriguez-Reinoso, F. (eds.) Introduction to Carbon Technologies, pp. 35-103. Universidad de Alicante, Alicante (1997)

Salvestrini, S., Sagliano, P., Iovino, P., Capass, S., Colella, C.: Atrazine adsorption by acid-activated zeolite-rich tuffs. Appl. Clay Sci. 49, 330-335 (2010)

Seredych, M., Hulicova-Jurcakova, D., Lu, G.Q., Bandosz, T.J.: Surface functional groups of carbons and the effects of their chemical character, density and accessibility to ions on electrochemical properties. Carbon 46, 1475-1488 (2008)

Stoeckli, F., Daguerre, E., Guillot, A.: The development of micropore volumes and widths during physical activation of various precursors. Carbon 37, 2075-2077 (1999)

Tang, W.W., Zeng, G.M., Gong, J.L., Liu, Y., Wang, X.Y., Liu, Y.Y., Liu, Z.-F., Chen, L., Zhang, X.R., Tu, D.Z.: Simultaneous adsorption of atrazine and $\mathrm{Cu}$ (II) from wastewater by magnetic multi-walled carbon nanotube. Chem. Eng. J. 211-212, 470-478 (2012)

Terzyk, A.P.: The impact of carbon surface composition on the diffusion and adsorption of paracetamol at different temperatures and at neutral pH. J. Colloid Interface Sci. 230, 219-222 (2000)

Wang, X.L., Sato, T., Xing, B.S.: Competitive sorption of pyrene on wood chars. Environ. Sci. Technol. 40, 3267-3272 (2006)

Weber, W.J., Morris, J.C.: Kinetics of adsorption of carbon from solutions. J. Sanit. Eng. Div. Am. Soc. Civ. Eng. 89, 31-63 (1963)

Welhouse, G.J., Bleam, W.F.: Atrazine hydrogen-bonding potentials. Environ. Sci. Technol. 27, 494-500 (1993)

Zhao, X., Ouyang, W., Hao, F., Lin, C., Wang, F., Han, S., Geng, X.: Properties comparison of biochars from corn straw with different pretreatment and sorption behaviour of atrazine. Bioresour. Technol. 147, 338-344 (2013) 